\title{
PREVENCIÓN DE LA CONDUCTA SUICIDA EN JÓVENES Y ADOLESCENTES: UNA REVISIÓN DESDE 1997
}

\section{SUICIDE BEHAVIOUR PREVENTION IN YOUTH AND ADOLESCENTS: A REVIEW SINCE 1997}

\author{
María Paz Mora Encinas \\ Psicóloga General Sanitaria
}

Cómo referenciar este artículo/How to reference this article:

Mora Encinas, M.P. (2018). Prevención de la Conducta Suicida en Jóvenes y Adolescentes: Una revisión desde 1997. Revista de Psicoterapia, 29(109), 203-226.

\begin{abstract}
Resumen
A pesar de que el suicidio constituye la tercera causa de muerte en jóvenes de entre 10 y 29 años, España carece de una guía de prevención específica para esa población, y la investigación realizada en nuestro país es muy escasa. Con el objetivo de proponer unas bases para el desarrollo de un modelo de prevención se realiza la presente revisión sistemática. Utilizando las bases de datos de PsycINFO, PsycArticles, MEDLINE, Psychology and Behavioral Sciences Collection y Web Of Science, se hallan 38 ensayos. Los estudios localizados muestran resultados prometedores de la Terapia de Solución de Problemas y las terapias familiares para la población indicada y selectiva; mientras que las intervenciones universales con mejores resultados son aquellas que inciden en el desarrollo de habilidades sociales y de afrontamiento desde un enfoque práctico. Los estudios sobre formación de personas capaces de reconocer un caso de riesgo arrojan datos contradictorios. Se demanda más investigación.

Palabras clave: jóvenes, adolescentes, conducta suicida, prevención, intervención.
\end{abstract}

\begin{abstract}
Although suicide ranks as the third leading cause of death in young people aged 10-29 years, Spain lacks a specific prevention guide for this population, and research in our country is very scarce. In order to propose a basis for the development of a prevention model, the present systematic review was conducted. Using the databases of PsycINFO, PsycArticles, MEDLINE, Psychology and Behavioral Sciences Collection and Web of Science, 38 trials were identified. These studies show promising outcomes from Problem Solving Therapy and family therapies as indicated and selected interventions. For universal interventions, the best results are those that impact on the development of social and coping skills. Studies on gatekeeper training yield contradictory data. More research is demanded.

Keywords: young, adolescents, suicidal behavior, prevention, intervention.
\end{abstract}




\section{INTRODUCCIÓN}

Según la Organización Mundial de la salud (OMS, 2017), el suicidio origina unas 800.000 muertes en el mundo cada año y es la segunda causa de defunción en jóvenes de entre 15 y 29 años. En España, según el Instituto Nacional de Estadística (INE), en 2015 (últimos datos registrados), el suicidio fue la segunda causa de muerte en la población de entre 20 y 29 años, produciendo aproximadamente el mismo número de muertes que los tumores. En poblaciones más jóvenes (15-19 años) el suicidio constituye la tercera causa de muerte, por debajo de los tumores y de los accidentes de tráfico, mientras que en edades más tempranas ocupa un séptimo puesto. A pesar de la gravedad que revisten estas cifras, pueden estar subestimadas, ya que se considera que parte de los accidentes de tráfico son suicidios encubiertos. Por otro lado, hay que tener en cuenta que estos datos contabilizan los suicidios exitosos. Se calcula que por cada suicidio consumado hay entre diez y veinte intentos (OMS, 2004).

El suicidio en la adolescencia se ha vinculado fuertemente con la presencia o historia de situaciones estresantes o traumáticas, como trastorno mental, enfermedad crónica dolorosa, historia de abuso, acoso escolar o problemas familiares (Navarro-Gómez, 2017), y con una carencia de recursos para hacerles frente, debida tanto a factores personales (desesperanza, hostilidad, impulsividad, escasa capacidad de resolución de problemas) como familiares o sociales.

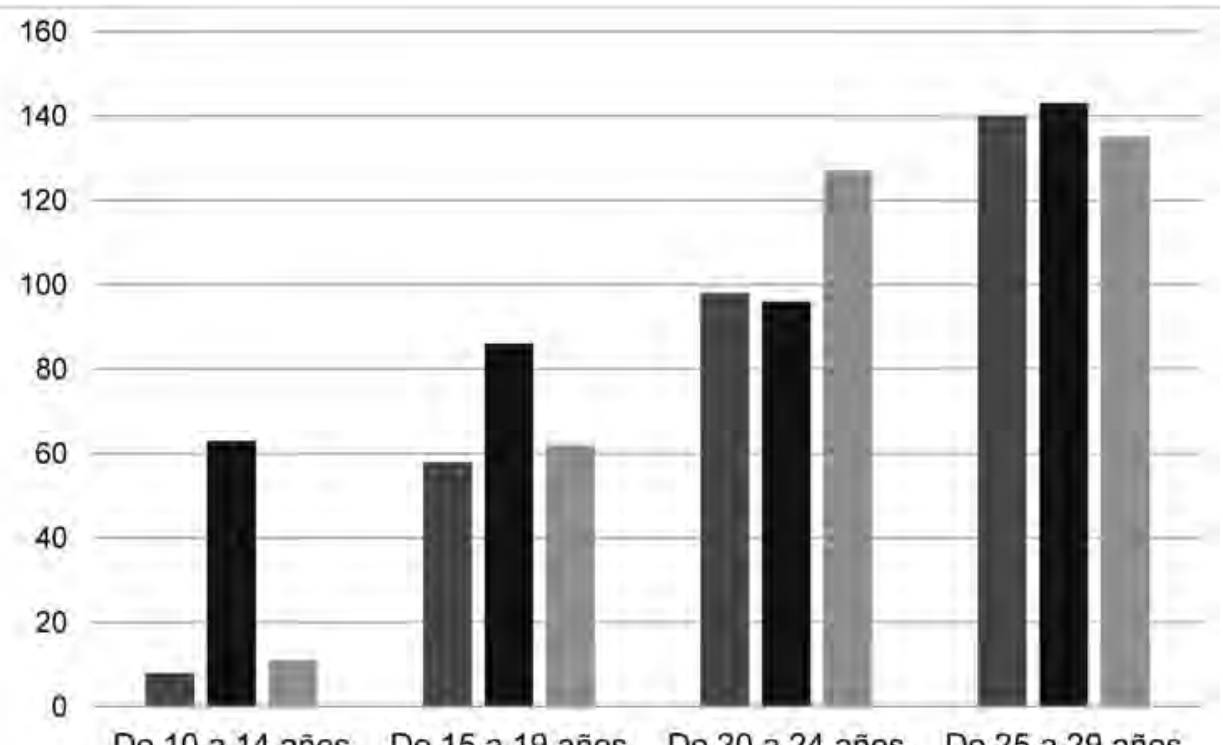

De 10 a 14 años De 15 a 19 años De 20 a 24 años De 25 a 29 años

Figura1. Frecuencia de las principales causas de muerte en España en 2015. Fuente INE. Elaboración propia 
La adolescencia es un periodo de transición entre la infancia y la adultez en el que los jóvenes deben adaptarse a los cambios psico-biológicos que experimentan y satisfacer con éxito las tareas vitales que son propias de ese momento, como pueden ser la consecución de la propia identidad y el inicio de las relaciones de pareja. Estos procesos, junto a las distorsiones cognitivas características de esta etapa (por ejemplo, la audiencia imaginaria o la fábula personal) y la gran importancia que cobran los pares y el sentirse aceptados por ellos, influyen en la autoestima y el autoconcepto. Es por esto una población vulnerable con recursos psicológicos todavía por desarrollar que, ante los desafíos que se pueda encontrar en la vida, puede sentirse sobrepasada y llegar a pensar en el suicidio como única solución. La fragilidad de esta etapa evolutiva queda reflejada en la tasa de suicidio, que se multiplica por cuatro en diez años (ver figura 2), de ahí la importancia de desarrollar un plan de prevención integral. Por otro lado, si se consigue que los jóvenes incorporen a su bagaje personal recursos, capacidad de solución de problemas y habilidades de afrontamiento frente a situación de estrés, a largo plazo, se conseguirá disminuir la tasa de suicidios en la población general.

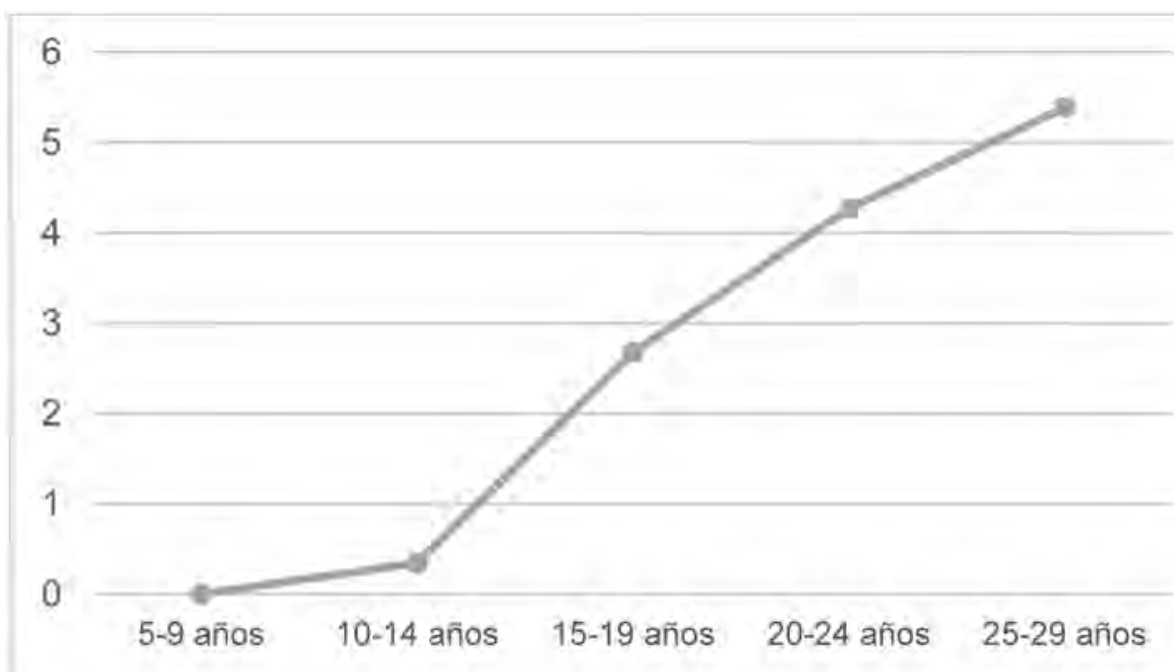

Figura 2. Tasa de suicidios por edad por cada 100000 jóvenes de cada grupo en España en 2015. Fuente INE. Elaboración propia.

A pesar de estas particularidades de la población adolescente y de que la OMS alerte sobre la situación del suicidio en los jóvenes, no existe en España una guía específica para esta población. La Guía de Práctica Clínica de Prevención y Tratamiento de la Conducta Suicida (GPC, Ministerio de sanidad, política social e igualdad, 2012) de España califica a esta población de riesgo y le dedica un apartado. Sin embargo, esto no parece suficiente. Resulta llamativo que, mientras 
existen guías de práctica clínica especializadas en esta población en el tratamiento de la obesidad, los trastornos del sueño o la Depresión Mayor, no lo haya ante la tercera causa de muerte en población juvenil.

En 2008 el Consejo de Europa publicó una resolución en la que demanda una mayor implicación de la investigación científica para prevenir el suicidio en niños y adolescentes. Sin embargo, la investigación sobre el suicidio en España es bastante escasa, más aún si hablamos de prevención en jóvenes. Las pocas publicaciones que se pueden encontrar suelen ser revisiones que contemplan la conducta suicida en adolescentes en su totalidad: epidemiología, factores de riesgo, de protección, etc., dedicando muy poco espacio a la prevención en sí. Existen estudios sobre programas preventivos en población adulta con resultados muy prometedores realizados desde el Hospital de la Santa Creu i Sant Pau (Tejedor, Díaz, Faus, Pérez y Solà, 2011; Farré et al., 2016), pero no se encuentra nada parecido realizado con población más joven.

Ante estas necesidades detectadas, se realiza la presente revisión bibliográfica con el fin de aportar unas bases desde las que se pueda articular una guía para la prevención del suicidio en jóvenes y de promover la investigación sobre la prevención del suicidio en este grupo etario en España.

\section{MÉTODO}

A la hora de localizar los artículos para esta revisión se establecieron los siguientes criterios de inclusión:

- deben tratar sobre la evaluación de intervenciones psicosociales (programas psicoterapéuticos, de psicoeducación, intervenciones en la comunidad, de apoyo, etc.) para el tratamiento o la prevención de la conducta suicida en adolescentes y jóvenes de entre 10 y 29 años.

- tipo diseños admitidos: experimentales y cuasi-experimentales

- tamaño muestral igual o mayor de 30.

- año de publicación: entre 1997 y 2016 ambos inclusive.

- los resultados pueden ser sobre: la conducta suicida, factores de riesgo, factores protectores, conocimientos, actitudes o competencias de «guardián».

Criterios de exclusión:

- estudios sobre aceptabilidad, viabilidad o niveles de implantación de las intervenciones

- estudios epidemiológicos sobre factores de riesgo o de protección

- reseñas de libros, revisiones, meta-análisis, descripciones o desarrollos de programas o protocolos

- estudios sin resultados

- estudios que tratan exclusiva o principalmente de conductas auto-lesivas sin intención suicida

- estudios referidos a intervenciones adaptadas a etnias específicas (por 
ejemplo: indios apaches de una reserva), cuyos resultados son difícilmente extrapolables a la población general española

- idiomas de publicación diferentes a: español, inglés, francés, italiano o portugués.

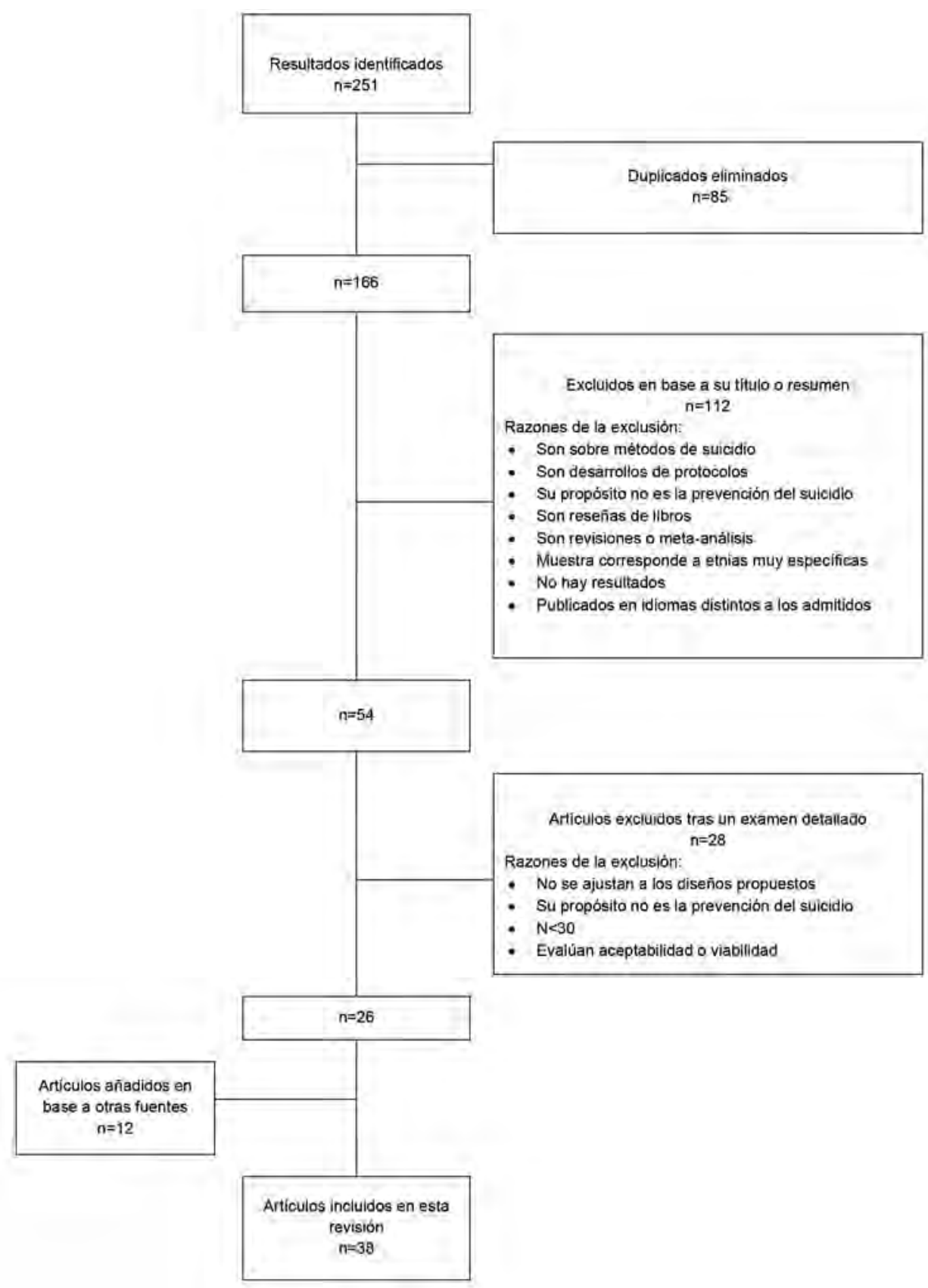

Figura3. Diagrama del proceso de identificación de los estudios 
El proceso de búsqueda se realizó utilizando las siguientes bases de datos: PsycINFO, PsycArticles, MEDLINE, PsychologyandBehavioral Sciences Collection y Web Of Science.

Las palabras clave utilizadas fueron: suicid* AND (preven* OR intervention OR treatment OR program OR therapy) AND (youth OR young OR adolescen* OR school OR college) estableciendo las siguientes restricciones:

- Texto completo en línea

- Publicado de 1997 a 2016

\section{RESULTADOS}

Se encontraron 251 resultados. Se quitaron 85 que estaban duplicados. De los 166 restantes 112 fueron eliminadas en base a su título o resumen según los criterios de inclusión y exclusión y, más adelante, tras un examen más detallado, se descartaron otros 28. Por último se añadieron 12 artículos identificados a través de revisiones previas o a través de algunos de los artículos descartados. De esta manera, finalmente 38 artículos forman parte de esta revisión.

Para su análisis se han examinado los estudios según su: estrategia de intervención, población diana, tamaño muestral, tipo de diseño, duración de la intervención, variables que se evalúan y resultados obtenidos. Para facilitar su lectura, cuando se haga mención a un estudio, aparecerá entre corchetes el número que tiene asignado en la Tabla 1 para su correcta identificación. 
Tabla 1.

Descripción de los estudios identificados

\begin{tabular}{|c|c|c|c|c|c|c|}
\hline Ref. & An̄o & Autores & Muestra & Disefio & $\begin{array}{l}\text { Caracteristicas de la inter. } \\
\text { vención }\end{array}$ & Variables \\
\hline 1 & 1998 & $\begin{array}{l}\text { Harrington } \\
\text { et al. }\end{array}$ & $\begin{array}{l}\text { Jóvenes menores } \\
\text { de } 16 \text { años con } \\
\text { intentos previos } \\
\text { de suicidio por } \\
\text { sobredosis } \\
\mathrm{N}=162\end{array}$ & ECA & $\begin{array}{l}4 \text { sesiones de Terapia de } \\
\text { Solucion de Problemas (TSP) } \\
\text { basada en la familia realizadas } \\
\text { en el hogar. } \\
\text { Grupo de control: cuidados } \\
\text { usuales. }\end{array}$ & $\begin{array}{l}\text { CS } \\
\text { FFR } \\
\text { FFP }\end{array}$ \\
\hline 2 & 1999 & $\begin{array}{l}\text { Davidson \& } \\
\text { Range }\end{array}$ & $\begin{array}{l}\text { Estudiante de } \\
\text { Magisterio que } \\
\text { finalizaban sus } \\
\text { estudios } \\
\mathrm{N}=75\end{array}$ & $\begin{array}{l}\text { Medidas } \\
\text { repelidas }\end{array}$ & $\begin{array}{l}\text { Formación en prevención del } \\
\text { súcidio de } 1 \text { hora }\end{array}$ & $\begin{array}{l}\text { Actitudes } \\
\text { CG }\end{array}$ \\
\hline 3 & 1999 & $\begin{array}{l}\text { Wiegersma, } \\
\text { Hofman \& Ziel- } \\
\text { huis }\end{array}$ & $\begin{array}{l}\text { Jóvenes (15-19 } \\
\text { años) } \\
\mathrm{N}=1361793\end{array}$ & $\begin{array}{l}\text { Casos y } \\
\text { controles }\end{array}$ & $\begin{array}{l}\text { Acceso libre y gratuito a con- } \\
\text { sulta de salud mental en las } \\
\text { instalaciones del colegio de } \\
1988 \text { a } 1993 \\
\text { Grupo de control: quienes no } \\
\text { lo tenian }\end{array}$ & CS \\
\hline 4 & 2000 & $\begin{array}{l}\text { Rotheram-Bo- } \\
\text { rus, Piacentini, } \\
\text { Cantwell, Belin, } \\
\text { \& Song }\end{array}$ & $\begin{array}{l}\text { Jóvenes en ries- } \\
\text { go }(12-18 \text { años) } \\
N=140 ; M=100 \%\end{array}$ & $\begin{array}{l}\text { Cuasi-ex- } \\
\text { perimental } \\
\text { de dos } \\
\text { grupos }\end{array}$ & $\begin{array}{l}1 \text { sesión de Intervención fami- } \\
\text { liar en el ámbito de urgencias } \\
\text { Grupo de control: cuidados } \\
\text { usuales }\end{array}$ & $\begin{array}{l}\text { CS } \\
\text { FFR }\end{array}$ \\
\hline 5 & 2001 & $\begin{array}{l}\text { Joiner. Voelz \& } \\
\text { Rudd }\end{array}$ & $\begin{array}{l}\text { Jövenes con } \\
\text { conducta suicida } \\
\text { cornórbida cón } \\
\text { Depresión Mayor } \\
\text { y/o trastorno de } \\
\text { ansiedad ( } 19-25 \\
\text { años) } \\
\mathrm{N}=44 ; \mathrm{M}=61 \%\end{array}$ & $\mathrm{ECA}$ & $\begin{array}{l}\text { Terapia de Solución de pro- } \\
\text { blemas intensiva ( } 9 \mathrm{~h} \text { al dia) } \\
\text { de tiempo limitado ( } 10 \text { dias) y } \\
\text { de tipo ambulatorio en grupo } \\
\text { ( } 8 \text { - } 14 \text { pers). } \\
\text { Grupo de control: cuidados } \\
\text { usuales }\end{array}$ & cs \\
\hline 6 & 2001 & $\begin{array}{l}\text { Thompsori } \\
\text { Eggert, Randell } \\
\text { \& Pike }\end{array}$ & $\begin{array}{l}\text { Jóvenes en ries- } \\
\text { go }(14-19 \text { afios) } \\
N=460 ; M=52 \%\end{array}$ & $E C A$ & $\begin{array}{l}\text { Intervención basada en la } \\
\text { escuela } \\
\text { Grupos experimentales: C-CA- } \\
\text { RE' o C-CARE + CAST }^{2} \\
\text { Grupo de control: cuidados } \\
\text { usuales }\end{array}$ & $\begin{array}{l}\text { CS } \\
\text { FFR } \\
\text { FFP } \\
\text { Actitudes }\end{array}$ \\
\hline
\end{tabular}

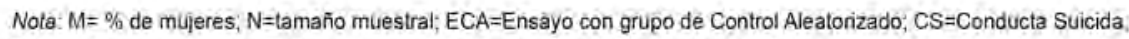
FFR=Factores de Riesgo; FFP=Factores de Protección: CG=Competencias de Guardián. 


\begin{tabular}{|c|c|c|c|c|c|c|}
\hline Ref. & Año & Autores & Muestra & Díseño & $\begin{array}{l}\text { Caracteristicas de la inter. } \\
\text { venciōn }\end{array}$ & Variables \\
\hline 7 & 2002 & $\begin{array}{l}\text { Eggert, Thomp- } \\
\text { son, Randell } \\
\text { \& Pik }\end{array}$ & $\begin{array}{l}\text { Jóvenes (14-19 } \\
\text { años) en riesgo } \\
\text { de abandono } \\
\text { escolar } \\
\mathrm{N}=341, \mathrm{M}=40 \%\end{array}$ & ECA & $\begin{array}{l}\text { Intervención basada en la } \\
\text { escuela } \\
\text { Grupos expérimentales: C-CA- } \\
\text { RE o C-CARE + CAST } \\
\text { Grupo de control: cuidados } \\
\text { usuales }\end{array}$ & $\begin{array}{l}\text { CS } \\
\text { FFR }\end{array}$ \\
\hline 8 & 2003 & $\begin{array}{l}\text { Stuart, Waalen } \\
\text { \& Haestromm }\end{array}$ & $\begin{array}{l}\text { Jóvenes }(12-14 \\
\text { años) } \\
\mathrm{N}=37\end{array}$ & $\begin{array}{l}\text { Medidas } \\
\text { repetidas }\end{array}$ & $\begin{array}{l}\text { Intervención basada en la } \\
\text { escuela: Many Helping Heart: } \\
\text { capacionación en guardia- } \\
\text { nes entre compañeros en } 2 \\
\text { sesiones de medio día y una } \\
\text { sesión de refresco } 3 \text { meses } \\
\text { más tarde. }\end{array}$ & $\begin{array}{l}\text { CG } \\
\text { Conocimiento } \\
\text { Actitudes }\end{array}$ \\
\hline 9 & 2004 & Huey et ail. & $\begin{array}{l}\text { Jóvenes ( } 10-17 \\
\text { anos) en riesgo } \\
N=156 ; M=35 \%\end{array}$ & ECA & $\begin{array}{l}\text { Terapia Multisistemica basada } \\
\text { en el hogar intensiva (diaria si } \\
\text { es necesario) de tiempo limita- } \\
\text { do ( } 3-6 \text { meses) }\end{array}$ & $\begin{array}{l}\text { CS: } \\
\text { FFR }\end{array}$ \\
\hline 10 & 2006 & $\begin{array}{l}\text { Portzky \& van } \\
\text { Heeringen }\end{array}$ & $\begin{array}{l}\text { Jóvenes }(14-18 \\
\text { años) } \\
N=172 ; M=63 \%\end{array}$ & $\begin{array}{l}\text { Disen̂o } \\
\text { Solomon }\end{array}$ & $\begin{array}{l}\text { Intervención basada en la } \\
\text { escuela } \\
\text { Sesión psicoeducativa de } 2 \\
\text { horas } \\
\text { Grupo de control no recibió } \\
\text { nada. }\end{array}$ & $\begin{array}{l}\text { FFR } \\
\text { FFP } \\
\text { Conocimiento } \\
\text { Actitudes }\end{array}$ \\
\hline 11 & 2007 & Ciffone & $\begin{array}{l}\text { Jóvenes (15-16 } \\
\text { años) } \\
\mathrm{N}=421\end{array}$ & ECA & $\begin{array}{l}\text { Intervención de psicoeduca- } \\
\text { ción basado en la escuela } \\
\text { Grupo de control: lista de } \\
\text { espera }\end{array}$ & Actitudes \\
\hline 12 & 2008 & $\begin{array}{l}\text { Cigularov. } \\
\text { Chen. Thurber } \\
\& \text { Stallones }\end{array}$ & $\begin{array}{l}\text { Jóvenes ( } 13-19 \\
\text { años) } \\
N=779 ; M=50 \%\end{array}$ & ECA & $\begin{array}{l}\text { Programa RAPP' de } 50.75 \\
\text { minutos insertado en el curri- } \\
\text { culo escolar }\end{array}$ & $\begin{array}{l}\text { Conocimiento } \\
\text { Actitudes } \\
\text { CG }\end{array}$ \\
\hline 13 & 2008 & $\begin{array}{l}\text { Eskin, Ertekin \& } \\
\text { Demir }\end{array}$ & $\begin{array}{l}\text { Jóvenes ( } 15-23 \\
\text { años) con Depre- } \\
\text { sión Mayor } \\
\mathrm{N}=46 ; \mathrm{M}=32 \%\end{array}$ & ECA & $\begin{array}{l}\text { TSP en } 6 \text { sesiones semanales } \\
\text { individuales de } 30 \text { a } 60 \mathrm{~min} \text {. } \\
\text { Grupo de control: lista de } \\
\text { espera }\end{array}$ & $\begin{array}{l}\text { CS } \\
\text { FFP }\end{array}$ \\
\hline 14 & 2008 & Wyman el al. & $\begin{array}{l}\text { Personal escolar } \\
\mathrm{N}=249\end{array}$ & ECA & $\begin{array}{l}\text { Formación en guardianes } \\
\text { (QPR) } \\
\text { Grupo de control: lista de } \\
\text { espera }\end{array}$ & $\begin{array}{l}\text { Conocimiento } \\
\text { Actitudes } \\
\text { CG }\end{array}$ \\
\hline 15 & 2009 & Baber $\&$ Bean & $\begin{array}{l}\text { Jóvenes ( } 14-15 \\
\text { ańos) y aduitos } \\
\mathrm{N}=300\end{array}$ & $\begin{array}{l}\text { Medidas } \\
\text { repetidas }\end{array}$ & Entrenamiento en guardianes & $\begin{array}{l}\text { Conocimiento } \\
\text { Actitudes } \\
\text { CG }\end{array}$ \\
\hline
\end{tabular}

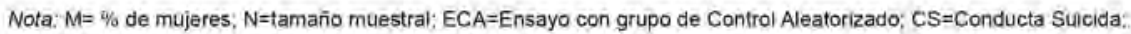
FFR=Factores de Riesgo; FFP =Faclores de Protección; CG=Competencias de Guardián 


\begin{tabular}{|c|c|c|c|c|c|c|}
\hline Ref. & Año & Autores & Muestra & Diseño & $\begin{array}{l}\text { Caracteristicas de la inter. } \\
\text { vención }\end{array}$ & Variables \\
\hline 16 & 2009 & Keller et al & $\begin{array}{l}\text { Adultos } \\
N=416 ; M=83,8 \%\end{array}$ & $\begin{array}{l}\text { Medidas } \\
\text { repetidas }\end{array}$ & Formación en guardianes QPR & $\begin{array}{l}\text { Conocimiento } \\
\text { Actitudes }\end{array}$ \\
\hline 17 & 2009 & Kinget al. & $\begin{array}{l}\text { Jóvenes (13-17 } \\
\text { años) en riesgo } \\
\mathrm{N}=448\end{array}$ & ECA & $\begin{array}{l}\text { YNST - } \mathrm{It}^{4} \text { (apoyo social) du- } \\
\text { rante } 3 \text { meses } \\
\text { Grupo de control: cuidados } \\
\text { usuales }\end{array}$ & $\begin{array}{l}\text { CS } \\
\text { FFR }\end{array}$ \\
\hline 18 & 2009 & $\begin{array}{l}\text { Klimes-Dou- } \\
\text { gan, Yuan, Lee, } \\
\text { \& Houri }\end{array}$ & $\begin{array}{l}\text { Jóvenes (14-18 } \\
\text { ańos) } \\
\mathrm{N}=426\end{array}$ & ECA & $\begin{array}{l}\text { Visualizar anunciós lelevisa- } \\
\text { dos o ver carteles con informa- } \\
\text { ción sobre el suicidio } \\
\text { Grupo de control: sin recibir } \\
\text { información }\end{array}$ & $\begin{array}{l}\text { Conocimiento } \\
\text { Actitudes } \\
\text { CG }\end{array}$ \\
\hline 19 & 2010 & Diamond el al & $\begin{array}{l}\text { Jóvenes }(12-17 \\
\text { años) en riesgo y } \\
\text { sus padres } \\
N \text { (jóvenes) }=66 . \\
\text { M(jóvenes) }=83 \%\end{array}$ & ECA & $\begin{array}{l}\text { Terapia Farniliar Basada en } \\
\text { el Apego (TFBA) durante } 3 \\
\text { meses }\end{array}$ & $\begin{array}{l}\text { CS } \\
\text { FFR }\end{array}$ \\
\hline 20 & 2010 & $\begin{array}{l}\text { Hooven, Hert- } \\
\text { ing. \& Snedker }\end{array}$ & $\begin{array}{l}\text { Jóvenes ( } 14-19 \\
\text { años) en riesgo y } \\
\text { sus padres } \\
\text { N(jovenes) }=593\end{array}$ & ECA & $\begin{array}{l}\text { Grupos experimentales: } \\
\text { C-CARE o P-CARE O C-CA- } \\
\text { RE+P-CARE } \\
\text { Grupo de control: intervención } \\
\text { minima }\end{array}$ & $\begin{array}{l}\text { CS } \\
\text { FFP } \\
\text { FFR }\end{array}$ \\
\hline 21 & 2010 & $\begin{array}{l}\text { Klimes-Dougan } \\
\text { \& Lee }\end{array}$ & $\begin{array}{l}\text { Jóvenes (18-35 } \\
\text { años) } \\
\mathrm{N}=279\end{array}$ & ECA & $\begin{array}{l}\text { Visualizar anuncios televisa- } \\
\text { dos o ver carteles con informa- } \\
\text { ción sobre el suicidio } \\
\text { Grupo de control: sin recibir } \\
\text { información }\end{array}$ & $\begin{array}{l}\text { Conocimiento } \\
\text { Actitudes } \\
\text { CG }\end{array}$ \\
\hline 22 & 2010 & Wyman et al. & $\begin{array}{l}\text { Jóvenes }(14-18 \\
\text { años) } \\
N=3128 ; M=54 \%\end{array}$ & $E C A$ & $\begin{array}{l}\text { Intervención basada en la } \\
\text { escuela: Sources of Strength }\end{array}$ & $\begin{array}{l}\text { CS } \\
\text { Actitudes } \\
\text { CG }\end{array}$ \\
\hline 23 & 2011 & Asarnow et al: & $\begin{array}{l}\text { Jóvenes ( } 14-18 \\
\text { años) en riesgo y } \\
\text { sus padres } \\
N=181: M=69 \%\end{array}$ & ECA & $\begin{array}{l}\text { Intervención familiar en el ám- } \\
\text { bito de urgencias: Family Inter- } \\
\text { vention for Suicide Prevention } \\
\text { (FISP) basada en la TCC en } \\
1 \text { sóla sesión más contactos } \\
\text { telefónicos durante un mes. } \\
\text { Grupo de control. cuidados } \\
\text { usuales }\end{array}$ & FFP \\
\hline 24 & 2011 & Cross et al & $\begin{array}{l}\text { Personal escolar } \\
\text { y padres } \\
N=147 ; M=87 \%\end{array}$ & ECA & $\begin{array}{l}\text { Formaciòn QPR más ensayos. } \\
\text { Grupo de control: Formación } \\
\text { QPR }\end{array}$ & $\begin{array}{l}\text { Conocimiento } \\
\text { CG }\end{array}$ \\
\hline
\end{tabular}

Nota: $\mathrm{M}=$ \% de mujeres; $\mathrm{N}=$ tamaño muestral; $E C A=$ Ensayo con grupo de Control Aleatorizado; $C S=C o n d u c t a$ Suicida: FFR $=$ Factores de Riesgo; FFP=Factores de Protección; $C G=$ Competencias de Guardián. 


\begin{tabular}{|c|c|c|c|c|c|c|}
\hline Ref. & Año & Autores & Muestra & Diseño & $\begin{array}{l}\text { Caracteristicas de la inter- } \\
\text { vención }\end{array}$ & Variables \\
\hline 25 & 2011 & $\begin{array}{l}\text { Esposi- } \\
\text { to-Smythers, } \\
\text { Spirito, Kahler, } \\
\text { Hunt \& Monti }\end{array}$ & $\begin{array}{l}\text { Jóvenes (13-17 } \\
\text { años) con con- } \\
\text { duota suicida y } \\
\text { abuso de canna- } \\
\text { bis o alcohol } \\
\mathrm{N}=40 ; \mathrm{M}=67 \%\end{array}$ & ECA & $\begin{array}{l}\text { Intervención familiar basada } \\
\text { en la Terapia Cognitivo-Con- } \\
\text { ductual (TCC). Sesiones } \\
\text { semanales durante } 6 \text { meses. } \\
\text { más sesiones quicenales } \\
\text { durante } 3 \text { meses, más } 3 \text { sesio- } \\
\text { nes mensuales }\end{array}$ & $\begin{array}{l}\text { CS } \\
\text { FFR }\end{array}$ \\
\hline 26 & 2011 & $\begin{array}{l}\text { King. Strunk \& } \\
\text { Sorter }\end{array}$ & $\begin{array}{l}\text { Jóvenes (14-18 } \\
\text { años) } \\
N=966 ; M=56 \%\end{array}$ & $\begin{array}{l}\text { Medidas } \\
\text { repetidas }\end{array}$ & $\begin{array}{l}\text { Intervención basada en la } \\
\text { escuela para la prevención } \\
\text { del suicidio y la depresión: } \\
\text { Surviving the Teens( }(8)^{6} \text { de } 4 \\
\text { sesiones de } 50 \text { min }\end{array}$ & $\begin{array}{l}\text { CS } \\
\text { FFR } \\
\text { Actitudes } \\
\text { CG }\end{array}$ \\
\hline 27 & 2012 & $\begin{array}{l}\text { Diamond. } \\
\text { Creed, Gillham, } \\
\text { Gallop \& Ha- } \\
\text { milton }\end{array}$ & $\begin{array}{l}\text { Jóvenes (13-17 } \\
\text { años) en riesgo y } \\
\text { sus padres } \\
\text { N(jovenes) }=66\end{array}$ & ECA & TFBA durante 12 semanas & $\begin{array}{l}\text { CS } \\
\text { FFR }\end{array}$ \\
\hline 28 & 2012 & $\begin{array}{l}\text { Hooven, Her- } \\
\text { ting. \& Snedker }\end{array}$ & $\begin{array}{l}\text { Jóvenes (14-19 } \\
\text { años) intentos } \\
\text { suicidas y ele- } \\
\text { vada ideación o } \\
\text { depresion, o } 2 \text { de } \\
\text { gstas } 3 \text { caracte- } \\
\text { risticas: mode- } \\
\text { rada depresión } \\
\text { e ideación con- } \\
\text { sumo de alcahol } \\
\text { y drogas; y sus } \\
\text { padres } \\
\mathrm{N}=615\end{array}$ & $E C A$ & $\begin{array}{l}\text { Grupos experimentales C-CA. } \\
\text { RE o P-CARE o C+P-CARE } \\
\text { C-CARE: } 2 \text { sesiones de } 2 \\
\text { horas } \\
\text { P-CARE: } 2 \text { sesiones de } 2 \\
\text { horas } \\
\text { Grupo de control: intervenciòn } \\
\text { usual }\end{array}$ & $\begin{array}{l}\text { CS } \\
\text { FFR } \\
\text { FFP }\end{array}$ \\
\hline 29 & 2012 & $\begin{array}{l}\text { Pasco, Wa- } \\
\text { llack, Sartin, \& } \\
\text { Dayton }\end{array}$ & $\begin{array}{l}\text { Jóvenes }(18-20 \\
\text { años) } \\
N=65 ; M=54 \%\end{array}$ & $\begin{array}{l}2 \text { grupos } \\
\text { con medi- } \\
\text { das pretest } \\
\text { y postest }\end{array}$ & $\begin{array}{l}\text { Formación en guardianes } \\
\text { Campus Connect de } 3 \text { horas } \\
\text { con psicoeducación y ejerci- } \\
\text { clos prácticos }\end{array}$ & $\mathrm{CG}$ \\
\hline 30 & 2012 & $\begin{array}{l}\text { Wharff, Ginnis, } \\
\text { \& Ross }\end{array}$ & $\begin{array}{l}\text { Jóvenes ( años) } \\
\text { en riesgo suicida } \\
\text { y sus padres } \\
\mathrm{N}=217\end{array}$ & $\begin{array}{l}\text { Cuasi-ex- } \\
\text { perimental } \\
\text { de cohorte }\end{array}$ & $\begin{array}{l}\text { Intervencióni de crisis basada } \\
\text { en la familià (ICBF) en una } \\
\text { sola sesión en el ámbito de } \\
\text { Urgencias (basado en la TCC. } \\
\text { TFM y TN) } \\
\text { Grupo de control cuidados } \\
\text { usuales }\end{array}$ & cs: \\
\hline
\end{tabular}

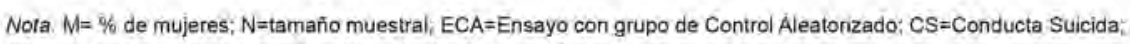
FFR=Factores de Riesgo; FFP=Factores de Protecciọn; CG=Competencias de Guardián. 


\begin{tabular}{|c|c|c|c|c|c|c|}
\hline Ref. & Año & Autores & Muestra & Diseño & $\begin{array}{l}\text { Caracteristicas de la inter- } \\
\text { vención }\end{array}$ & Variables \\
\hline 31 & 2013 & $\begin{array}{l}\text { Pineda \& Da- } \\
\text { dds }\end{array}$ & $\begin{array}{l}\text { Jóvenes }(12-17 \\
\text { años) en riesgo y } \\
\text { sus padres } \\
\text { N(jovenes) }=48 \\
\text { M(jóvenes) }=75 \%\end{array}$ & ECA & $\begin{array}{l}\text { Intervención familiar: RAP.P' } \\
4 \text { sesiones semanales de } 2 \\
\text { horas sumado al tratamiento } \\
\text { con el joven. } \\
\text { Grupo de control: cuidados } \\
\text { usuales }\end{array}$ & $\begin{array}{l}\text { CS } \\
\text { FFR }\end{array}$ \\
\hline 32 & 2014 & Cimini et al. & $\begin{array}{l}\text { Jóvenes universi- } \\
\text { tarios y personal } \\
\text { de la universidad } \\
N=335 ; M=51 \%\end{array}$ & $\begin{array}{l}\text { Medidas } \\
\text { repetidas }\end{array}$ & $\begin{array}{l}\text { Capacitación de guardianes } \\
\text { de } 1,5 \text { horas }\end{array}$ & $\begin{array}{l}\text { Conocimiento } \\
\text { CG }\end{array}$ \\
\hline 33 & 2015 & $\begin{array}{l}\text { Asarnow, Berk. } \\
\text { Hughes, \& } \\
\text { Anderson }\end{array}$ & $\begin{array}{l}\text { Jóvenes ( } 11-18 \\
\text { ańos) en riesga y } \\
\text { sus padres } \\
N=35 ; M=86 \%\end{array}$ & $\begin{array}{l}\text { Medidas } \\
\text { repetidas }\end{array}$ & $\begin{array}{l}\text { Programa SAFETY: interven- } \\
\text { ción familiar basada en la TCC } \\
\text { de } 12 \text { semanas de duracion. }\end{array}$ & $\begin{array}{l}\text { CS } \\
\text { FFR }\end{array}$ \\
\hline 34 & 2015 & King et at & $\begin{array}{l}\text { Jóvenes ( } 18-28 \\
\text { años) que cum- } \\
\text { plen } 2 \text { criterios } \\
\text { de :ideación } \\
\text { suicida, } 2 \text { inten- } \\
\text { tos de suicidio; } \\
\text { depresión y abu- } \\
\text { so de alcohol. } \\
\mathrm{N}=60 ; \mathrm{M}=59 \%\end{array}$ & ECA & $\begin{array}{l}\text { Programa online eBridge } \\
\text { basado en la entrevista mo- } \\
\text { tivacional durante } 2 \text { meses y } \\
\text { feedback del cribado. } \\
\text { Grupo de control: feedback del } \\
\text { cribado. }\end{array}$ & $\begin{array}{l}\text { CS } \\
\text { FFP }\end{array}$ \\
\hline 35 & 2015 & $\begin{array}{l}\text { Silverstone } \\
\text { et al. }\end{array}$ & $\begin{array}{l}\text { Jóveries ( } 11-19 \\
\text { años) con y sin } \\
\text { riesgo } \\
N=2790 ; M=48 \%\end{array}$ & $\begin{array}{l}2 \text { diseños: } \\
\text { con grupo } \\
\text { de control } \\
\text { sin alea- } \\
\text { torización } \\
\text { y medidas } \\
\text { repetidas }\end{array}$ & $\begin{array}{l}\text { Intervención en la escuela } \\
\text { basada en la TCC: Programa } \\
\text { EMPATHY" }\end{array}$ & $\begin{array}{l}\text { CS } \\
\text { FFR } \\
\text { FFP }\end{array}$ \\
\hline 36 & 2015 & $\begin{array}{l}\text { Walrath, Ga- } \\
\text { rraza, Reid. } \\
\text { Goldston \& } \\
\text { McKeon }\end{array}$ & $\begin{array}{l}\text { (No aparece } \\
\text { información so- } \\
\text { bre las personas } \\
\text { que recibieron la } \\
\text { intervención) } \\
\text { Evaluación: Jóve- } \\
\text { nes (10-24 añas). } \\
N>500.000\end{array}$ & $\begin{array}{l}\text { cuasi-ex. } \\
\text { perimental } \\
\text { con grupo } \\
\text { de control }\end{array}$ & $\begin{array}{l}\text { Entrenamiento en guardiàn } \\
\text { (de } 1 \text { hora a pocas dias) en } \\
\text { varias poblaciones } \\
\text { Grupo de control poblaciones } \\
\text { sin entrenamiento. }\end{array}$ & $\mathrm{CS}$ \\
\hline
\end{tabular}

Nota: $\mathrm{M}=\%$ de mujeres; $\mathrm{N}=$ tamaño muestral; $\mathrm{ECA}=$ Ensayo con grupo de Control Aleatorizado, $\mathrm{CS}=\mathrm{Conducta}$ Sujcida: FFR=Factores de Riesgo; FFP=Factores de Protección; CG=Competencias de Guardián. 


\begin{tabular}{|c|c|c|c|c|c|c|}
\hline Ref. & Año & Autores & Muestra & Diseño & $\begin{array}{l}\text { Caracteristicas de la inter. } \\
\text { vención }\end{array}$ & Variables \\
\hline 37 & 2015 & Wasserman & $\begin{array}{l}\text { Jóvenes (14-15 } \\
\text { años) } \\
\mathrm{N}=11111\end{array}$ & ECA & $\begin{array}{l}\text { Proyecto SEYLE: se comparan } \\
4 \text { intervenciones en la escuela: } \\
\text { concienciación }{ }^{10} \text {, formación de } \\
\text { guardianes para el profesora- } \\
\text { do, cribado de adolescentes } \\
\text { en riesgo e intervención mini- } \\
\text { ma, durante } 4 \text { semanas }\end{array}$ & $\mathrm{CS}$ \\
\hline 38 & 2016 & $\begin{array}{l}\text { Ghoncheh., } \\
\text { Gould, Twisk, } \\
\text { Kerkhof. \& Koot }\end{array}$ & $\begin{array}{l}\text { Aduiltos }(21.62 \\
\text { años) } \\
N=190: M=8.6 \%\end{array}$ & ECA & $\begin{array}{l}\text { Formación de guardianes onli- } \\
\text { ne:Mental Health Online } \\
\text { Grupo de control: lista de } \\
\text { espera }\end{array}$ & $\begin{array}{l}\text { Conocimiento } \\
\text { CG }\end{array}$ \\
\hline
\end{tabular}

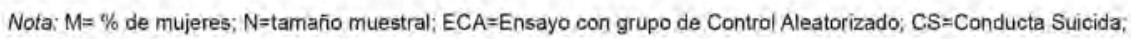
FFR=Factores de Riesgo; FFP=Factores de Protección; CG=Competencias de Guardián.

1. C-CARE (Counselor-CARE), programa basado en el aprendizaje social. Realizado de manera individual (3.5-4 horas), consta de los siguientes elementos: información, evaluación, feedback, apoyo y motivación. fortalecimiento de habilidades sociales y de afrontamiento, promoción de la conducta de búsqueda de ayuda y coordinación con padres y colegio.

2. CAST (Coping And Support Training): programa de reforzamiento del C-CARE. Son 12 sesiones bisemanaies de una hora.

? RAPP (Raising Awareness of Personal Power) programa psicoeducativo sobre la depresión, el trastorno bipolar y las sef̂ales de alarma del suicidia, pautas de acción ante un caso de riesgo y lugares de ayuda. El métado se basa en lecturas. juegos interactivos, role-play y análisis de historias.

4 YNST-II (Youth Nominated Support Team Version II): programa basado en el apoyo social. Un adulto elegido por el joven (con la aceptación de los padres) proveerá apoyo emocional, feedback e informaciôn durante los tres meses posteriores a su hospitalización.

3 P-CARE (Parent-CARE): Programa complementario al C-CARE dirigido a padres, cuyos objetivos son mejorar el manejo y resolución de conflictos, aumentar la escucha activa y las muestras de apoyo, y aprender a guiar a sus hijos en la adquisición de habilidades de automanejo.

- Surviving the Teens $\circledast$, programa basado en el modelo de autoeficacia de Bandura, provee a los jóvenes de información sobre suicidio y depresión, y estrategias efectivas de afrontamiento frente a los estresores de la vida.

7 RAP.P (Resourceful Adolescent Parent Program): programa complementario al tratamiento usual del adolescente que involucra a los padres facilitándoles información sobre los servicios adecuados de ayuda y el desarrollo normal del adolescente a la vez que les proporciona estrategias para ayudar a sus hijos a manejar el conflicto y las situaciones de estrés. identificando las fortalezas de los padres.

- SAFETY (Safe Alternatives for Teens \& Youth): intervención familiar que parte de un análisis cognitivo-conductual de la conducta suicida desde una perspectiva ecológica y con la implicación de toda la familia en el desarrollo de un plan "de seguridad", Basado en la TCC y la TMS.

a EMPATHY (Empowering a Multimodal Pathway Towards Healthy Youth); programa a varios niveles que incluye: cribado general, TCC a población universal para incrementar la resilencia a la depresión, y una entrevista seguida TCC on-line para la población en ríesgo.

10. Programa de Concienciación del SEYLE (Saving and Empowering Young Lives in Europe): Programa cognitivo-emocional-experiencial. A través de lecturas, debates y role-plays se plantean a los jóvenes problemas relacionados con la salud mental y sẹ les enseña a desarrollar habilidades sociales y de solución de problemas y a identificar cuándo deben ser utilizadas. 


\section{Tipos de estrategias}

- Tratamientos individuales [5, 13, 17 y 34$]$ ya sea a través de un formato individual, en grupo o vía internet. Tipos de intervención: Terapia de Solución de problemas, terapias basadas en el apoyo social y en la Terapia Cognitivo-Conductual (TCC).

- Intervenciones familiares [1, 4, 9, 19, 20, 23, 25, 27, 28, 30, 31 y 33]: se interviene de una manera integrada sobre el joven, sus padres y el funcionamiento familiar Las intervenciones encontradas están basadas en: la TCC, la Terapia Familiar Sistémica (TFS), Terapia Familiar Basada en el Apego (TFBA), en el desarrollo de habilidades sociales y autoeficacia, o en la psicoeducación.

- Intervenciones en el ámbito escolar [3, 5, 7, 8, 10, 11, 12, 22, 26, 29, 32, 35 y 37] engloban un conjunto variado de propuestas que se desarrollan en la escuela. Pueden ser intervenciones universales o indicadas. Se identifican tres grupos: enfocados a aumentar los factores de protección y disminuir los de riesgo; formación de los propios jóvenes como «guardianes» de sus compañeros (psicoeducación, adquisición de habilidades de guardián, etc.); e intervenciones en el ambiente.

- Capacitación $[2,14,15,16,29,36$ y 38]: Dirigidos a adultos de distintos ámbitos, son programas psicoeducativos sobre el suicidio (tasas de suicidio, signos de alarma, etc.) y de consecución de habilidades (mejorar la comunicación con los jóvenes, aprender a abordar el tema del suicidio con ellos, reconocer señales de alarma, etc). Algunos de ellos utilizan el entrenamiento Question Persuade Refer (QPR, Quinnett, 1995). Muchos de estas intervenciones reciben el nombre de «formación de guardianes» (gatekeeper training).

- Basadas en la publicidad [18 y 21]: buscan hallar la eficacia de anuncios televisados o carteles informativos sobre el suicidio.

\section{Población diana y tamaño muestral}

La edad de los jóvenes sobre los que se realizan estos estudios oscila entre los 10 y los 25 años, aunque en un estudio realizado sobre universitarios se llega a alcanzar los 35 [21].

Tanto las intervenciones de carácter individual como las familiares son de tipo indicado o selectivo con presencia de intentos previos de suicidios, significativa ideación suicida, alta puntuación en síntomas depresivos, abuso de sustancias, etc. Los criterios de exclusión más habituales suelen ser: síntomas psicóticos o bipolares y estados que limiten la función cognitiva. Se observa una distribución por sexos con una presencia de mujeres superior al $55 \%$, excepto en dos estudios, en los que se presenta en torno al $35 \%[9,13]$.

Las estrategias en el ámbito escolar, basadas en la publicidad y de capacitación realizan intervenciones universales (excepto dos de las realizadas en la 
escuela [6,7]). Esto concuerda con un tamaño muestral mayor y con una distribución de la participación por sexos más igualada (proporción de chicas de un 48$63 \%$ ). De este modo, mientras que la mediana de los tamaños muestrales de las estrategas individuales y familiares es 61 y 148 respectivamente, la del resto de estrategias es superior a 215, llegando a los 460 en el caso de las intervenciones en la escuela.

Por otro lado, es importante señalar que, aunque las intervenciones basadas en la capacitación se intervenga principalmente sobre los adultos, las evaluaciones pueden estar realizadas sobre ellos o sobre los jóvenes sobre los que aquellos puedan entrar en contacto [36].

\section{Tipo de diseño}

La investigación de Silverstone et al. [35] está proyectada de un modo complejo en el que un subgrupo de la muestra participa en un diseño cuasiexperimental con grupo de control a la vez que todo el conjunto de la muestra forma parte de un diseño de medidas repetidas. Pasco et al. [29] no especifica si la asignación al grupo experimental o al grupo de control se ha realizado experimentalmente. De los estudios restantes, siete son de medidas repetidas $[2,8,15,16,26$, 32 y 33], cinco cuasi-experimentales con grupo de control [3, 4, 30 y 36$]$ y el resto son ensayos con grupo de control aleatorizado.

\section{Duración de la intervención}

Atendiendo a su mediana, las intervenciones que muestran mayor duración en el tiempo son las basadas en una estrategia basada en la familia (alrededor de 3 meses), seguidas de las estrategias basadas en el individuo (cerca de los dos meses).

Las intervenciones en el ámbito escolar tienen una duración variada. Así, si la intervención se basa en cambios ambientales (por ejemplo, existencia de una consulta de acceso libre), la intervención puede extenderse hasta los dos años. En el caso de tratarse de sesiones o cursos psicoeducativos la duración oscila entre una $\mathrm{y}$ cuatro sesiones de entre una y dos.

Por otro lado, las intervenciones en capacitación suelen ser breves, desde una hora a pocos días. Aunque las más rápidas son aquellas que estudian el impacto de la publicidad (minutos).

\section{Variables}

Las variables dependientes están en función del sujeto evaluado:

- Jóvenes: conducta suicida, síntomas depresivos, consumo de sustancias, estilo de afrontamiento, conocimiento sobre el suicidio, actitud hacia el suicidio, actitud de ayuda, autoeficacia.

- Padres: conocimiento sobre el suicidio, actitud hacia el suicidio, actitud de ayuda, autoeficacia en sus competencias para distinguir un caso de riesgo y afrontarlo, conducta promotora de apego, estrés y depresión 
parental y funcionamiento familiar.

- Adultos «guardianes»: conocimiento sobre el suicidio, actitud hacia el suicidio, actitud de ayuda, autoeficacia.

Mientras que las intervenciones sobre poblaciones selectivas o indicadas sí evalúan la conducta suicida, las universales casi nunca tienen en cuenta el efecto sobre esta variable. De hecho, de los veinte estudios de enfoque universal, solamente seis la incluyen [3, 22, 26, 36, 35 y 37].

Los estudios centrados en el individuo y en la familia examinan principalmente la conducta suicida, y, en menor medida, factores de riesgos y factores de protección.

De los 13 ensayos que investigan programas basados en la escuela, sólo siete (dos de ellos en población indicada [6 y 7]) valoran el efecto sobre la conducta suicida [3, 22, 26, 35 y 37$]$; cinco evalúan factores de riesgo [5, 7, 10, 26 y 35$]$; tres, factores de protección $[5,10,35]$; siete, las actitudes sobre el suicido $[5,8,10,11$, 12,22 y 26]; cinco, el conocimiento [8, 10, 12 y 32]; y seis, la conducta de guardián o las competencias para ayudar $[8,12,22,26,29$ y 32$]$.

Los programas de capacitación tienen como objetivo la mejora en las competencias para ayudar, el conocimiento y las actitudes sobre el suicidio; excepto en el caso de Walrath et al. [36], que mide el impacto de los guardianes en la tasa de suicidio entre los jóvenes.

En los estudios sobre el impacto de la publicidad se estudia el cambio de actitud, el conocimiento y la intención de búsqueda de ayuda.

\section{Resultados de las intervenciones}

\section{Conducta suicida}

El suicidio consumado sólo se evalúa en estudios de carácter epidemiológico, como el de Walrath et al. [36] y el de Wiegersma et al. [3]. En el primer caso se halló que disminuía significativamente la tasa de suicidios en jóvenes de entre 10 y 24 años en aquellas poblaciones donde se había entrenado a guardianes. Sin embargo, al cabo de dos años esas diferencias desaparecían. Por su parte el estudio epidemiológico de Wiegersma et al. no obtuvo diferencias entre los lugares con acceso a consulta de salud gratuita frente a los que no lo tenían.

La TSP $[5,13]$ resulta eficaz tanto en reducir la ideación, como los planes, escritos y amenazas suicidas. Las tentativas suicidas no fueron evaluadas en estos trabajos.

Las intervenciones familiares en general resultan más eficaces en reducir las tentativas y las amenazas planes y escritos que la ideación [9 y 25]. Destacan, con resultados significativamente mejores sobre todas las variables de la conducta suicida, los programas: C-CARE, P-C-CARE [20, 28], RAP-P [31] y el SAFETY [33]. El TFBA [19 y 27] arroja resultados prometedores, pero sólo evalúan ideación. En el ámbito escolar muy pocas intervenciones evalúan su efecto sobre la 
conducta suicida. Destacan la investigación de King et al. [26] y, sobre todo, el proyecto SEYLE [37] en su variante de «concienciación», consiguiendo una reducción de los intentos en más de un $50 \%$ con un tamaño muestral de más de 11000 jóvenes y un seguimiento de 12 meses.

\section{Factores de riesgo}

Según los estudios examinados, los programas C-CARE, P-C-CARE [20, 28], TCC familiar [25], RAP-P [31] y SAFETY [33]; los basados en la TSP [13]; los escolares Surviving the Teens ${ }^{\circledR}$ [26] y EMPATHY [35] destacan al reducir los factores de riesgo como depresión, desesperanza, ansiedad, ira, consumo de sustancias y/o depresión parental.

\section{Factores de protección}

El programa C-CARE+CAST resultó beneficioso en el ámbito escolar con un seguimiento de seis meses en el aumento de la capacidad de solución de problemas [6 y 7$]$.

Los programas C-CARE y P-C-CARE incrementaron significativamente la autoeficacia de los jóvenes y que los resultados se mantenían durante seis años [20 y 28].

La TSP fue superior a la lista de espera en jóvenes con depresión mayor para aumentar los niveles de asertividad y autoestima con un seguimiento de 12 meses [13].

Tanto la plataforma on-line eBridge []34, como el programa escolar Sources of Strength [22]consiguieron un significativo aumento de la conducta de búsqueda de ayuda frente al grupo de control. Un anuncio televisado sobre la prevención del suicidio aumenta la intención de buscar ayuda entre universitarios frente a los que vieron un cartel o no recibieron información [21]. Sin embargo, entre adolescentes de alto riesgo, aquellos que vieron el anuncio televisado o los carteles mostraron peor afrontamiento [18].

\section{Competencias de guardián}

A pesar de encontrarse mejoras en su autoeficacia como «guardianes» tras realizar el programa QPR (Quinnett, 1995) [14, 16 y 38], no se encontraron diferencias significativas en un cambio de conducta (preguntar a jóvenes, etc.) con respecto a la lista de espera, o entre el programa QPR y QPR+ensayos [12 y 18 respectivamente].

\section{Conocimiento sobre el suicidio}

El programa QPR demostró que incrementaba significativamente tanto el conocimiento real como el percibido [12 y 38].

El anuncio televisado aumentó significativamente el conocimiento en jóvenes adolescentes frente a las condiciones de cartel o no información. En universitarios no se halló ningún efecto [18 y 21]. 


\section{Actitudes sobre el suicidio}

Tanto los programas de capacitación [2, 12 y 16], como los escolares [8, 11, $12,22$ y 26$]$ pueden mejorar significativamente las actitudes sobre el suicidio.

\section{DISCUSIÓN}

Tras el repaso de la bibliografía que se ha expuesto, se pueden distinguir tres tipos fundamentales de estrategias en función del tipo de población a las que van dirigidas: las intervenciones indicadas o selectivas, las universales y la estrategia dirigida a la identificación de casos de riesgo: la formación de «guardianes».

Respecto al primer caso, se pueden hallar intervenciones focalizadas en el joven o en el conjunto familiar. Los resultados en general son positivos, sobre todo si se tiene en cuenta que el grupo de control suele recibir los «cuidados usuales» y que la mayoría consigue mejoras significativas en la disminución de la ideación suicida y/o de las tentativas. Por ese motivo, una fuente de información importante puede ser examinar qué hay de diferente en las que no han mostrado esos resultados.

Una de las intervenciones en las que no se ha evidenciado una mejora con respecto al grupo de control ha sido la basada en el apoyo social [17]. Los autores justifican su programa aduciendo que se han encontrado diferencias en los niveles de apoyo familiar encontrado entre jóvenes suicidas y no suicidas. Pero, teniendo en cuenta los resultados obtenidos en este ensayo (y en otro anterior realizado por los mismo autores) y los obtenidos en las intervenciones familiares, parece que lo que realmente es significativo para los jóvenes es percibir el apoyo por parte de sus propios familiares, de su grupo de apoyo natural y primario; y no de otro adulto, aunque lo hayan elegido ellos mismos. De hecho, las intervenciones familiares que han conseguido resultados positivos tienen en común la reconstrucción o el fortalecimiento del tejido de las relaciones familiares, la identificación de sus fortalezas como familia, el desarrollo de habilidades de comunicación y la adquisición de estrategias para afrontar conflictos y problemas.

En esta revisión han aparecido dos intervenciones familiares que, aun persiguiendo los mismo objetivos que las otras, no han conseguido buenos resultados. Son aquellas realizadas en una sesión en el ámbito de la atención en urgencias [4 y 23]. Por lo que queda claro que una sola sesión para toda la familia es insuficiente, a pesar de que haya contactos telefónicos durante un mes.

Las intervenciones individuales que han obtenido buenos resultados en la disminución de la conducta suicida estaban basadas en la TSP [5 y 13], con un seguimiento de un año. Otras revisiones, como la de Calear et al. (2015), que consiguió localizar cuatro ensayos sobre TSP (no familiar), encuentran resultados contradictorios.

En el estudio que acabamos de mencionar, Calear et al. sacan algunas conclusiones interesantes, como que, mientras las intervenciones individuales disminuyen la ideación suicida, las familiares y grupales rebajan la frecuencia de los intentos suicidas. Sin embargo, la presente revisión no puede confirmar esas 
afirmaciones, ni tampoco otras que hacían referidas a la variación en la ideación o las tentativas en función de si se medían las variables tras un seguimiento corto o largo (superior a los 18 meses).

Mención aparte merece la entrevista motivacional on-line eBridge [34], que sólo buscaba promover la búsqueda de ayuda y obtuvo resultados positivos en ese sentido, aun a pesar de no lograr una alta adherencia. Tampoco es baladí este efecto, puesto que, aunque algunos programas producen un impacto positiva sobre esta variable, no todos lo hacen e incluso hay evidencia de consecuencias iatrogénicas (Klimes-Dougan, Klingbeil y Melle, 2013). Las investigaciones de Klimes-Dougan y su equipo sobre los efectos de la publicidad dan cuenta de estos efectos negativos, aunque, según muestra, estos están mediados por la edad y la pertenencia al grupo de riesgo suicida o no. Así, mientras que entre los 14 y 18 años, la publicidad puede disminuir la conducta de búsqueda de ayuda en poblaciones en riesgo, este efecto no aparece con un grupo de jóvenes en riesgo también pero más mayores (18-35 años) [18 y 32].

En lo referido a las intervenciones universales y a los programas de capacitación, se echa en falta una evaluación del cambio en la conducta suicida. Algo que también ponen de manifiesto otras revisiones (GPC, 2012; Katzet al., 2013; Miller, Eckert y Mazza, 2009). Al leerlos, surgen las preguntas: ¿es suficiente que haya un aumento significativo en el conocimiento del suicidio o en el cambio de actitudes para que disminuyan los suicidios?, ¿es suficiente tener personas significativamente mejor preparadas para reconocer un caso de riesgo?

Sólo hay un ensayo que examine al mismo tiempo conducta suicida y cambio de actitudes: King et al., en su estudio sobre el programa Surviving the teens ${ }^{\circledR}[26]$, evaluó al mismo tiempo la conducta suicida, la depresión y desesperanza, el cambio en las actitudes y la intención de ayuda a un compañero, obteniendo resultados significativamente positivos en todas las variables con respecto a la línea base. Sin embargo, su programa abarcaba diversos puntos (estrategias de afrontamiento y psicoeducación) por lo que no podemos deducir qué es lo que en definitiva baja los índices de ideación y tentativas.

Hay otro artículo que aporta algunas claves al respecto: Walrath et al. [36] comparan los índices de suicidio de las poblaciones donde se ha formado a guardianes con aquellos en los que no (equiparando variables que pudieran desvirtuar los resultados). Los resultados son parcialmente positivos, ya que, aunque un año tras los cursos de formación disminuían los suicidios, y esta disminución correlacionaba con el número de personas capacitadas; al cabo de los dos años estos efectos desaparecían por completo. La tarea que se presenta, por lo tanto, es descubrir a qué se debe esta disminución del efecto. No parece lógico pensar que se deba a una disminución de los conocimientos o la autoeficacia de los guardianes, ya que, con la práctica, estas habilidades suelen aumentar. Habría que investigar si existen diferencias en la motivación que influyan en su conducta, o qué otros factores pueden estar interviniendo. 
Por otro lado, los datos aportados por Walrath et al. entran en contradicción con los resultados del proyecto SEYLE [37]. En este último se pusieron en marcha cuatro programas durante cuatro semanas: cribado, formación de guardianes para el profesorado (programa QPR), programa de concienciación para los alumnos y una intervención mínima (consistente en proporcionar información de contacto de servicios de salud y de grupos de vida saludable y en colgar de carteles alertando contra el suicidio). Con un año de seguimiento, los resultados del programa QPR no mostraron diferencias en la conducta suicida. Los autores apuntan como una posible explicación que los guardianes sólo pueden actuar ante conductas accesibles a la observación y muchas veces la ideación suicida es disimulada.

A pesar de la escasez de investigaciones en población universal que evalúen la conducta suicida, se han encontrado tres que han mostrado éxito en esta variable. Los factores comunes de estos tres programas son estar diseñados para enseñar a los alumnos a desarrollar habilidades sociales y de afrontamiento. Una de ellos es el programa de concienciación del proyecto SEYLE. De hecho, el éxito de este programa fue mayor que otros programas de intervención en salud pública sobre prevención de acoso escolar o de consumo de tabaco, ya que se observó un porcentaje de descenso mucho mayor (del 50\% con respecto al $14-23 \%$ de estos otros programas). Los otros dos programas (con un seguimiento menor, sólo de tres meses), son el Surviving the Teens ${ }^{\circledR}$ y el EMPATHY [26 y 35 respectivamente]. Lo interesante del programa EMPATHY es que, tras un cribado inicial, sólo recibieron la TCC un grupo de 151 alumnos considerados de alto riesgo y todos los estudiantes de séptimo y octavo curso (dejando fuera a los alumnos de los grados sexto, noveno, décimo, undécimo y duodécimo), y, sin embargo, se observó un descenso significativo general en depresión y riesgo suicida al volver a evaluar a un total de 2790 alumnos. Lo que sugiere que se haya producido una especia de «contagio positivo» entre los alumnos.

Queda manifiesto que es necesaria una mayor investigación sobre este campo, en particular el ámbito español, que permita comprobar la eficacia tanto de las intervenciones como de sus componentes, a corto y a largo plazo sobre las variables de interés (principalmente conducta suicida). A lo largo de la investigación que ha supuesto este trabajo se han detectados ensayos en los que se daba cuenta de intervenciones adaptadas a las necesidades culturales y sociales de poblaciones muy específicas consideradas de alto riesgo (tribus nativas americanas, comunidad latina americana, comunidad afro-americana urbana, etc.), que se descartaron debido a que se consideró que las muy concretas circunstancias de estos grupos eran difícilmente extrapolables a la población general española. También se ha visto cómo la diferencia de edad influye en los efectos de una intervención (aun dentro de una población joven), y que un criterio de exclusión que aparece repetidamente es presentar síntomas psicóticos (a pesar de que es un factor de riesgo para el suicidio). Todo ello invita a pensar en que, según vaya avanzando al investigación, sea necesario ir desarrollando intervenciones adaptadas a grupos de población con 
unas características concretas, más allá de la distinción entre intervención indicada, selectiva o universal.

\section{Limitaciones}

La revisión ha partido de artículos que se encontraran en línea, por lo que, aunque por medio de la misma bibliografia analizada se ha intentado localizar otros artículos de interés, es más que seguro que datos valiosos hayan quedado fuera de este trabajo. Por otro lado, por cuestiones de extensión, no se han incluido revisiones sistemáticas y meta-análisis hallados, aunque se han comentado sus hallazgos más sobresalientes en la discusión. Otra limitación está causada por los mismos ensayos, y es que, excepto en la frecuencia de suicidios consumados o en el número de ingresos que contabiliza el hospital, la mayoría de los datos son conseguidos mediante pruebas auto-informe.

\section{CONCLUSIONES}

Tras lo expuesto, se propone como punto de arranque un modelo de prevención en población juvenil apoyado en tres pilares.

1. Formación de guardianes efectiva que permita detectar un caso de riesgo antes de que se produzca un intento.

2. Intervenciones indicadas y selectivas basada en la TSP y en la terapia familiar

3. Intervenciones universales insertadas en la escuela que provean a los estudiantes de herramientas de afrontamiento y de solución de problemas, con un fuerte componente práctico, y adaptadas a las situaciones en las que se pueda ver envuelta la población adolescente.

\section{Nota del autor}

A José María Calés, mi tutor del Trabajo Fin de Máster, quien tan bien me supo guiar y motivar para que este trabajo llegara a buen fin. 


\section{Referencias bibliográficas}

*Asarnow, J. R., Baraff, L. J., Berk, M. S., Grob, C., Devich-Navarro, M., Suddath, R., Piacentini, J., RotheramBorus, M. J., Cohen, D. y Tang, L. (2011). Effects of an Emergency Department mental health intervention for linking pediatric suicidal patients to follow-up mental health treatment: a randomized controlled trial. Psychiatr Serv., 62(11), 1303-1309. Recuperado de: https://www.ncbi.nlm.nih.gov/pmc/articles/ PMC3251923/

*Asarnow, J. R., Berk, M., Hughes, J. L., y Anderson, N. L. (2015). The SAFETY program: A treatmentdevelopment trial of a cognitive-behavioral family treatment for adolescent suicide attempters. Journal of Clinical Child and Adolescent Psychology, 44(1), 194-203. Recuperado de: https://www.ncbi.nlm.nih.gov/ pubmed/25255931

*Baber, K., y Bean, G. (2009). Frameworks: A community-based approach to preventing youth suicide. Journal of Community Psychology, 37(6), 684-696. Recuperado de: www.theconnectprogram.org/sites/default/ files/site-content/docs/Frameworks-Acommunitybasedapproachtopreventingyouthsuicide.pdf

Calear, A. L., Christensen, H., Freeman, A., Fenton, K., Busby Grant, J., van Spijker, B., Donker, T. (2015). A systematic review of psychosocial suicide prevention interventions for youth. European Child \& Adolescent Psychiatry, 25(5), 467-482.

*Ciffone, J. (2007). Suicide prevention: An analysis and replication of a curriculum-based high school program. Social Work, 52(1), 41-49.

*Cigularov, K., Chen, P., Thurber, B. W., y Stallones, L. (2008). Investigation of the effectiveness of a school-based suicide education program using three methodological approaches. Psychological Services, 5(3), 262-274. Recuperado de: https://www.researchgate.net/profile/Konstantin_Cigularov/publication/ 232591119_Investigation_of_the_Effectiveness_of_a_School-Based_Suicide_Education_Program_Using Three_Methodological_Approaches/links/558ab60e-08ae48b7b56d8939.pdf

*Cimini, M. D., Rivero, E. M., Bernier, J. E., Stanley, J. A., Murray, A. D., Anderson, D. A., , Wright, H. R. y Bapat, M. (2014). Implementing an audience-specific small-group gatekeeper training program to respond to suicide risk among college students: A case study. Journal of American College Health, 62(2), 92-100.

Consejo de Europa (2008). Resolución 1608 Child and teenage suicide in Europe: a serious public health issue. Recuperado de: http://assembly.coe.int/nw/xml/XRef/Xref-XML2HTML-en.asp?fileid=17639\&lang=en

*Cross, W. F., Seaburn, D., Gibbs, D., Schmeelk-Cone, K., White, A. M. y, Caine E. D. (2011). Does Practice Make Perfect? A Randomized Control Trial of Behavioral Rehearsal on Suicide Prevention Gatekeeper Skills. Journal of Primary Prevention, 32(3-4), 195-211. Recuperado de: https://www.ncbi.nlm.nih.gov/pubmed/ 21814869

*Davidson, M. W., y Range, L. M. (1999). Are teachers of children and young adolescents responsive to suicide prevention training modules? Yes. Death Studies, 23(1), 61-71.

*Diamond, G. S., Creed, T., Gillham, J., Gallop, R., y Hamilton, J. L. (2012). Sexual trauma history does not moderate treatment outcome in attachment-based family therapy (ABFT) for adolescents with suicide ideation. Journal of Family Psychology, 26(4), 595-605. Recuperado de: https://www.ncbi.nlm.nih.gov/ pubmed/22709259

*Diamond, G. S., Wintersteen, M. B., Brown, G. K., Diamond, G. M., Gallop, R., Shelef, K., y Levy, S. (2010). Attachment-based family therapy for adolescents with suicidal ideation: a randomized controlled trial. Department of Psychiatry and Human Behavior Faculty Papers, paper 8. Recuperado de: http:// jdc.jefferson.edu/phbfp/8

*Eggert, L. L., Thompson, E. A., Randell, B. P., y Pik, K. C. (2002). Preliminary effects of brief school-based prevention approaches for reducing youth suicide-risk behaviors, depression, and drug involvement. Journal of Child and Adolescent Psychiatic Nursing, 15(2), 48-64.

*Eskin, M., Ertekin, K., y Demir, H. (2008). Efficacy of a problem-solving therapy for depression and suicide potential in adolescents and young adults. Cognitive Therapy and Research, 32(2), 227-245. Recuperado de: www.academia.edu/download/46824419/Efficacy_of_a_Problem-Solving_Therapy_fo2016062727035-eeq9sq.pdf

*Esposito-Smythers, C., Spirito, A., Kahler, C. W., Hunt, J., y Monti, P. (2011). Treatment of co-occurring substance abuse and suicidality among adolescents: A randomized trial. Journal of Consulting and Clinical Psychology, 79(6), 728-739. Recuperado de: https://www.ncbi.nlm.nih.gov/pubmed/22004303

Eurostat. (2015) Crude death rate by suicide of Young people by sex and age. Recuperado de: http://appsso.eurostat.ec.europa.eu/nui/submitViewTableAction.do 
Eurostat. (2015) Suicide death rate by age group. Recuperado de: http://ec.europa.eu/eurostat/web/productsdatasets/-/tsdph240

Farré, A., Portella, M. J., De Angel, L., Díaz, A., Diego-Adeliño, J., Vegué J., Durán-Sindreu, S., Faus, G., Tejedor, C., Álvarez, E. y Pérez, V. (2016). Benefits of a secondary prevention program in suicide. A 1-year followup experimental study compared with a treatment-as-usual control group. Crisis, 37(4), 281-289.

*Ghoncheh, R., Gould, M. S., Twisk, J. W., Kerkhof, A. J., y Koot, H. M. (2016). Efficacy of adolescent suicide prevention E-learning modules for gatekeepers: A randomized controlled trial. JMIR Mental Health, 3(1), e8. Recuperado de: https://www.ncbi.nlm.nih.gov/pubmed/26825006

Instituto Nacional de Estadística (2017). Defunciones por causas (lista reducida), sexo y edad. Recuperado de: http://www.ine.es/jaxi/Tabla.htm?path=/t15/p417/a2015/10/\&file=01001.px\&L=0

Instituto Nacional de Estadística (2017). Tasa de mortalidad por causas (lista reducida), sexo y edad. Recuperado de: http://www.ine.es/jaxi/Tabla.htm?path=/t15/p417/a2015/10/\&file=01004.px\&L=0

Hacker, K., Collins, J., Gross-Young, L., Almeida, S., y Burke, N. (2008). Coping with youth suicide and overdose: One community's efforts to investigate, intervene, and prevent suicide contagion. Crisis: The Journal of Crisis Intervention and Suicide Prevention, 29(2), 86-95.

*Harrington, R., Kerfoot, M., Dyer, E., McNiven, F., Gill, J., Harrington, V., Woodham, A. M. y Byford, S. (1998). The home-based family intervention resulted in reduced suicidal ideation only for patients without major depression. J. Am. Acad. Child Adolesc. Psychiatry, 37(5), 512-518. Recuperado de: https:// pdfs.semanticscholar.org/8d6f/6e557a770c2fbdd865c84f095a48d46365ad.pdf

*Hooven, C., Herting, J. R. y Snedker, K. A. (2010). Long-term outcomes for the Promoting CARE Suicide Prevention Program. Am J Health Behav. 34(6), 721-736. Recuperado de: https://www.ncbi.nlm.nih.gov/ pubmed/20604697

*Hooven, C., Herting, J. R. y Snedker, K. A. (2012). Promoting CARE: including parents in youth suicide prevention. Fam Community Health, 35(3), 225-235. Recuperado de: https://www.ncbi.nlm.nih.gov/ pubmed/22617413

*Huey, S. J., Henggeler, S. W., Rowland, M. D., Halliday-Boykins, C. A., Cunningham, P. B., y Pickrel, S. G. (2004). Multisystemic therapy effects on attempted suicide by youths presenting psychiatric emergencies. J. Am. Acad. Child Adolesc. Psychiatry, 43(2), 183-190. Recuperado de: http://www-bcf.usc.edu/ hueyjr/ MST_effects_on_attempted_suicide.pdf

*Joiner, T. E. J., Voelz, Z. R., y Rudd, M. D. (2001). For suicidal young adults with comorbid depressive and anxiety disorders, problem-solving treatment may be better than treatment as usual. Professional Psychology: Research and Practice, 32(3), 278-282.

Katz, C., Bolton, S., Katz, L. Y., Isaak, C., Tilston-Jones, T., y Sareen, J. (2013). A systematic review of schoolbased suicide prevention programs. Depression and Anxiety, 30(10), 1030-1045.

*Keller, D. P., Schut, L. J., Puddy, R. W., Williams, L., Stephens, R. L., McKeon, R., y Chermack, S. (2009). Tennessee lives count: Statewide gatekeeper training for youth suicide prevention. Professional Psychology: Research and Practice, 40(2), 126-133. Recuperado de: https://www.researchgate.net/profile/ Robert_Stephens2/publication/224123991_Tennessee_Lives_Count_Statewide_Gatekeeper_Training for_Youth_Suicide_Prevention/links/54b54-1dfb0cf26833efd0ace7.pdf

*King, C. A., Eisenberg, D., Zheng, K., Czyz, E., Kramer, A., Horwitz, A., et al. (2015). Online suicide risk screening and intervention with college students: A pilot randomized controlled trial. Journal of Consulting and Clinical Psychology, 83(3), 630-636. Recuperado de: https://www.ncbi.nlm.nih.gov/pubmed/25688811

*King, C. A., Klaus, N., Kramer, A., Venkataraman, S., Quinlan, P., y Gillespie, B. (2009). The youth-nominated support Team-Version II for suicidal adolescents: A randomized controlled intervention trial. Journal of Consulting and Clinical Psychology, 77(5), 880-893. Recuperado de: https://www.ncbi.nlm.nih.gov/ pubmed/19803568

*King, K. A., Strunk, C. M., y Sorter, M. T. (2011). Preliminary effectiveness of surviving the teens(®) suicide prevention and depression awareness program on adolescents' suicidality and self-efficacy in performing help-seeking behaviors. Journal of School Health, 81(9), 581-590.

Klimes-Dougan, B., Klingbeil, D. A., y Meller, S. J. (2013). The impact of universal suicide-prevention programs on the help-seeking attitudes and behaviors of youths. Crisis: The Journal of Crisis Intervention and Suicide Prevention, 34(2), 82-97. 
*Klimes-Dougan, B., y Lee, C. S. (2010). Suicide prevention public service announcements: Perceptions of young adults. Crisis: The Journal of Crisis Intervention and Suicide Prevention, 31(5), 247-254. Recuperado de: https://www.researchgate.net/profile/Bonnie_Klimes-Dougan/publication/49661371_ Suicide_Prevention_Public_Service_Announcements_Perceptions_of_Young_Adults/links/ $53 \mathrm{f35fe} 70 \mathrm{cf} 2 \mathrm{dd} 48950 \overline{\mathrm{cb}} 3 \mathrm{af} /$ Suicide-Prevention-Public-Service-Announcements-Perceptions-of-YoungAdults.pdf

*Klimes-Dougan, B., Yuan, C., Lee, S., y Houri, A. K. (2009). Suicide prevention with adolescents: Considering potential benefits and untoward effects of public service announcements. Crisis: The Journal of Crisis Intervention and Suicide Prevention, 30(3), 128-135. Recuperado de: http://www.save.org/wp-content/ uploads/2016/08/ContentServer-2.pdf

Miller, D. N., Eckert, T. L., y Mazza, J. J. (2009). Suicide prevention programs in the schools: A review and public health perspective. School Psychology Review, 38(2), 168-188. Recuperado de: http:// knoxvillepsychs.pbworks.com/f/Suicide+Prevention+Programs+in+the+Schools-2009.pdf

Ministerio de sanidad, política social e igualdad (2012). Guía de Práctica Clínica de Prevención y Tratamiento de la Conducta Suicida. Galicia: Avalia-t. Recuperado de: http://www.guiasalud.es/GPC/GPC_481_ Conducta_Suicida_Avaliat_compl.pdf

Nrugham, L., Herrestad, H., y Mehlum, L. (2010). Suicidality among norwegian youth: Review of research on risk factors and interventions. Nordic Journal of Psychiatry, 64(5), 317-326.

Organización Mundial de la Salud (2004). El suicidio, un problema de salud pública enorme y sin embargo prevenible, según la OMS. Recuperado de: http://www.who.int/mediacentre/news/releases/2004/pr61/es/

Organización Mundial de la Salud (2017). Suicidio. Nota descriptiva. Recuperado de: http://www.who.int/ mediacentre/factsheets/fs398/es/

*Pasco, S., Wallack, C., Sartin, R. M., \& Dayton, R. (2012). The impact of experiential exercises on communication and relational skills in a suicide prevention gatekeeper-training program for college resident advisors. Journal of American College Health, 60(2), 134-140.

*Pineda, J., \& Dadds, M.R. (2013). Family intervention for adolescents with suicidal behavior: a randomized controlled trial and mediation analysis. Journal of the American Academy of Child and Adolescent Psychiatry, 52(8), 851-862. doi: 10.1016/j.jaac.2013.05.015.

*Portzky, G., y van Heeringen, K. (2006). Suicide prevention in adolescents: A controlled study of the effectiveness of a school-based psycho-educational program. Journal of Child Psychology and Psychiatry, 47(9), 910918.

*Rotheram-Borus, M. J., Piacentini, J., Cantwell, C., Belin, T. R., y Song J. (2000). The 18-month impact of an Emergency Room intervention for adolescent female suicide attempters. Journal of Consulting and Clinical Psychology, 68(6), 1081-1093. Recuperado de: http://citeseerx.ist.psu.edu/viewdoc/download? doi=10.1.1.386.5127\&rep=rep1\&type $=$ pdf

*Silverstone, P. H., Bercov, M., Suen, V. Y. M., Allen, A., Cribben, I., Goodrick, J., et al. (2015). Initial findings from a novel school-based program, EMPATHY, which may help reduce depression and suicidality in youth. Plos One, 10(5), e0125527-e0125527. Recuperado de: https://www.ncbi.nlm.nih.gov/pmc/articles/ PMC4431804/

Spirito, A., Stanton, C., Donaldson, D., \& Boergers, J. (2002). Treatment-as-usual for adolescent suicide attempters: Implications for the choice of comparison groups in psychotherapy research. Journal of Clinical Child and Adolescent Psychology, 31(1), 41-47. Recuperado de: https://www.researchgate.net/publication/ 11514944_Treatment-as-Usual_for_Adolescent_Suicide_Attempters_Implications_for_the_ Choice_of_Comparison_Groups_in_Psychotherapy_Research

*Stuart, C., Waalen, J. K., y Haestromm, E. (2003). Many helping hearts: an evaluation of peer gatekeeper training in suicide risk assessment. Death Studies, 27,321-333. Recuperado de: https:/www.google.es/url?sa=t\&rct= $\mathrm{j} \& \mathrm{q}=\&$ esrc $=\mathrm{s} \&$ source $=$ web\& $\mathrm{cd}=2 \& \mathrm{cad}=\mathrm{rja} \& u a c t=8 \& \mathrm{ved}=0 \mathrm{ahUKEwj} 6 \mathrm{nY}$ XuKzXAhWBKcAKHQDDfgQFggwMAE\&url=https $\% 3 \mathrm{~A} \% 2 \mathrm{~F} \% 2 \mathrm{Fmsrc}$.fsu.edu $\% 2$ Fsystem $\% 2 \mathrm{Ffiles} \% 2 \mathrm{FStuart} \% 2520 \mathrm{et} \% 2520 \mathrm{al}$ $\% 25202003 \% 2520$ Many $\% 2520$ helping $\% 2520$ hearts— $\% 2520$ an $\% 2520$ evaluation $\% 2520$ of $\% 2520$ peer $\% 2520$ gatekeeper $\% 2520$ training\%2520in $\% 2520$ suicide $\% 2520$ risk\%2520assessment.pdf \&usg=AOvVaw2PK-Tf3VyoaqtpbtBvBBDk

Tejedor, C., Díaz, A., Faus, G., Pérez, V., y Solà, I. (2011). Resultados del programa de prevención de la conducta suicida. Distrito de la Dreta de l'Eixample de Barcelona. Actas Esp Psiquiatría, 39(05), 280-7. Recuperado de: https://www.actaspsiquiatria.es/repositorio/13/73/ESP/13-73-ESP-280-287-385438.pdf 
*Thompson, E. A., Eggert, L. L., Randell, B. P., \& Pike, K. C. (2001). Evaluation of indicated suicide risk prevention approaches for potential high school dropouts. American Journal of Public Health, 91(5), 742752. Recuperado de: https://www.ncbi.nlm.nih.gov/pmc/articles/PMC1446664/

*Walrath, C., Garraza, L. G., Reid, H., Goldston, D. B., y McKeon, R. (2015). Impact of the garrett lee smith youth suicide prevention program on suicide mortality. American Journal of Public Health, 105(5), 986-993. Recuperado de: https://www.ncbi.nlm.nih.gov/pubmed/25790418

*Wasserman, D. (2015). Outcomes of the SEYLE proyect on school-based suicide prevention Mental health of European youth. Meeting of the Group of Governmental Experts on Mental Health and Well-Being. Recuperado de:

http://ec.europa.eu/transparency/regexpert/index.cfm?do=groupDetail.groupDetailDoc\&id=17384\&no=5

*Wharff, E. A., Ginnis, K. M., y Ross, A. M. (2012). Family-based crisis intervention with suicidal adolescents in the emergency room: A pilot study. Social Work, 57(2), 133-143. Recuperado de: https://academic.oup.com/ sw/article-abstract/57/2/133/1936666?redirectedFrom=PDF

*Wiegersma, P. A., Hofman, A., y Zielhuis, G. A. (1999). Prevention of suicide by youth health care. Public Health, 113(3), 125-130. Recuperado de: http://www.rug.nl/research/portal/files/3171528/Wiegersma 1999_Public_Health.pdf

*Wyman, P. A., Brown, C. H., Inman, J., Cross, W., Schmeelk-Cone, K., Guo, J. y Pena, J. B. (2008). Randomized trial of a gatekeeper program for suicide prevention: 1-year impact on secondary school staff. Journal of Consulting and Clinical Psychology, 76(1), 104-115. Recuperado de: https://www.ncbi.nlm.nih.gov/ pubmed/18229988

*Wyman, P. A., Brown, C. H., LoMurray, M., Schmeelk-Cone, K., Petrova, M., Yu, Q., Walsh, E. y Wang, W. (2010). An outcome evaluation of the sources of strength suicide prevention program delivered by adolescent peer leaders in high schools. American Journal of Public Health, 100(9), 1653-1661. Recuperado de: https://www.ncbi.nlm.nih.gov/pubmed/20634440 